Acta Crystallographica Section B

Structural

Science

ISSN 0108-7681

\title{
The crystal and molecular structure of $o$-phenanthroline
}

S. Nishigaki, H. Yoshioka and K. Nakatsu

This electronic document was scanned from an archival copy of material deposited to accompany a paper published in an IUCr journal. In many cases the only accessible copy was a microfilm of a poor-quality original. 


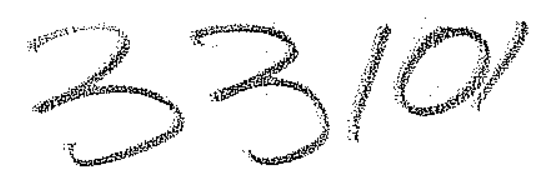

Thermal parameters and their es.a." $\left(A^{2} \times 10^{3}\right.$ for $N$ and $\left.C\right)$

The temperature factor is of the form

\begin{tabular}{|c|c|c|c|c|c|c|}
\hline$N(1)$ & $\operatorname{los}_{11} \frac{(150}{60(3)}$ & $\frac{y_{2} 2}{74(3)}$ & $\begin{array}{l}\frac{1}{33} \\
65(3)\end{array}$ & $\begin{array}{l}\frac{112}{-4(3)} \\
-y^{2}\end{array}$ & $\begin{array}{r}-23 \\
6(3)\end{array}$ & $\begin{array}{l}0 \\
51 \\
5(2)\end{array}$ \\
\hline C( 2$)$ & $84(4)$ & $83(6)$ & $68(4)$ & $-1(4)$ & $4(4)$ & $4(3)$ \\
\hline$C(3)$ & $130(6)$ & $78(6)$ & $75(4)$ & $-7(5)$ & $-2(5)$ & H1 14$)$ \\
\hline $\mathrm{C}(4)$ & 135461 & $62(5)$ & $82(5)$ & $-2(5)$ & $-6(5)$ & $46(5)$ \\
\hline $\mathrm{C}(\mathrm{s})$ & 9593 & 05063 & $162(7)$ & $0(5)$ & $2(6)$ & $6:(5)$ \\
\hline$c(6)$ & $55(4)$ & $67(6)$ & $182(s)$ & $5(4)$ & $17(6)$ & $45(4)$ \\
\hline $\mathrm{CO} 73$ & $82(5)$ & $108(B)$ & $157(7)$ & $-22(6)$ & $42(7)$ & $-40(5)$ \\
\hline $\mathrm{C}(\mathrm{B})$ & $156(\mathrm{~B})$ & $108(7)$ & $95(5)$ & $-46(7)$ & $21(7)$ & $-42(5)$ \\
\hline$C(0)$ & $132(6)$ & 7560 & $75(5)$ & $-9(5)$ & $-2(5)$ & $-5(4)$ \\
\hline$N(10)$ & $B Z(3)$ & $87(4)$ & $60(3)$ & $3(4)$ & $10(4)$ & $5(3)$ \\
\hline$C(1])$ & $58(4)$ & $56(5)$ & $81(4)$ & $2(4)$ & $I 7(4)$ & $I(3)$ \\
\hline$C(2)$ & $59(4)$ & $56(4)$ & $76(4)$ & $-5(3)$ & $8(4)$ & $5(3)$ \\
\hline$C(13)$ & $76(4)$ & $51(5)$ & $110(5)$ & $-2(4)$ & $-2(4)$ & $42(4)$ \\
\hline$C(14)$ & $67(4)$ & $70(5)$ & $116(5)$ & $-11(4)$ & $22(5)$ & $-8(4)$ \\
\hline$N(1+3$ & $68(4)$ & $69(4)$ & $83(4)$ & $6(4)$ & $9(4)$ & $28(5)$ \\
\hline$C\left(2^{11}\right)$ & $64(4)$ & $97(6)$ & $92(5)$ & $2 \pi(5)$ & $35(5)$ & $32(4)$ \\
\hline$C(5)$ & $75(4)$ & $121(7)$ & $91(5)$ & $29(6)$ & $20(6)$ & $18(4)$ \\
\hline$C\left(4^{\prime}\right)$ & $82(5)$ & $124(8)$ & $B I(5)$ & $38(6)$ & $-1,(5)$ & $19(4)$ \\
\hline$c(5)$ & $70(5)$ & $68(5)$ & $93(5)$ & $B(5)$ & $-14(4)$ & $-1(4)$ \\
\hline$C(127)$ & $35(3)$ & $62(4)$ & $69(3)$ & $-2(4)$ & $-I(4)$ & $2(2)$ \\
\hline$C\left(13^{\prime}\right)$ & $52(4)$ & $74(5)$ & $77(4)$ & $4(4)$ & $-4(4)$ & $3(3)$ \\
\hline $\mathrm{H}(2)$ & $0.06(2)$ & & & & & \\
\hline$A(3)$ & $0.08(2)$ & & & & & \\
\hline$B(4)$ & $0.07(2)$ & & & & & \\
\hline$H(5)$ & $0.06(2)$ & & & & & औ \\
\hline 備( 6$)$ & $0.07(2)$ & & & & & \\
\hline H( 7$)$ & $0.12(3)$ & & & & & \\
\hline$E(B)$ & $0.09(3)$ & & & & & \\
\hline $9(9)$ & $0.06(2)$ & & & & & \\
\hline
\end{tabular}

$+127 y 006(2)$

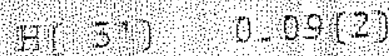

$(1,4) \div 0.06(2)$

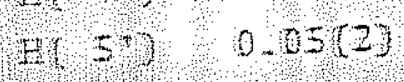


Goserved and calculated structure ampitudes (sn botans $x$ lo) for o-phonathroline
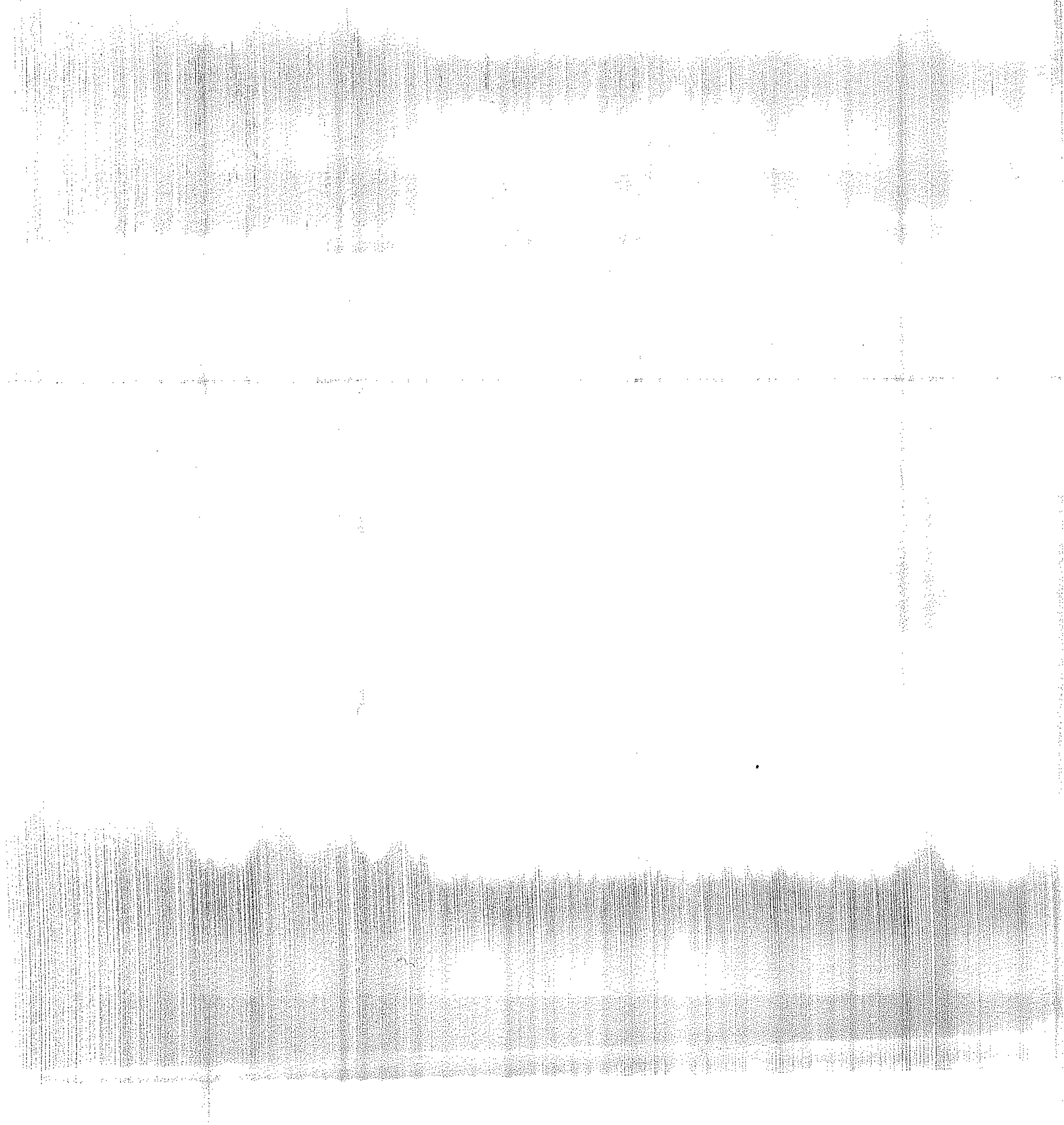


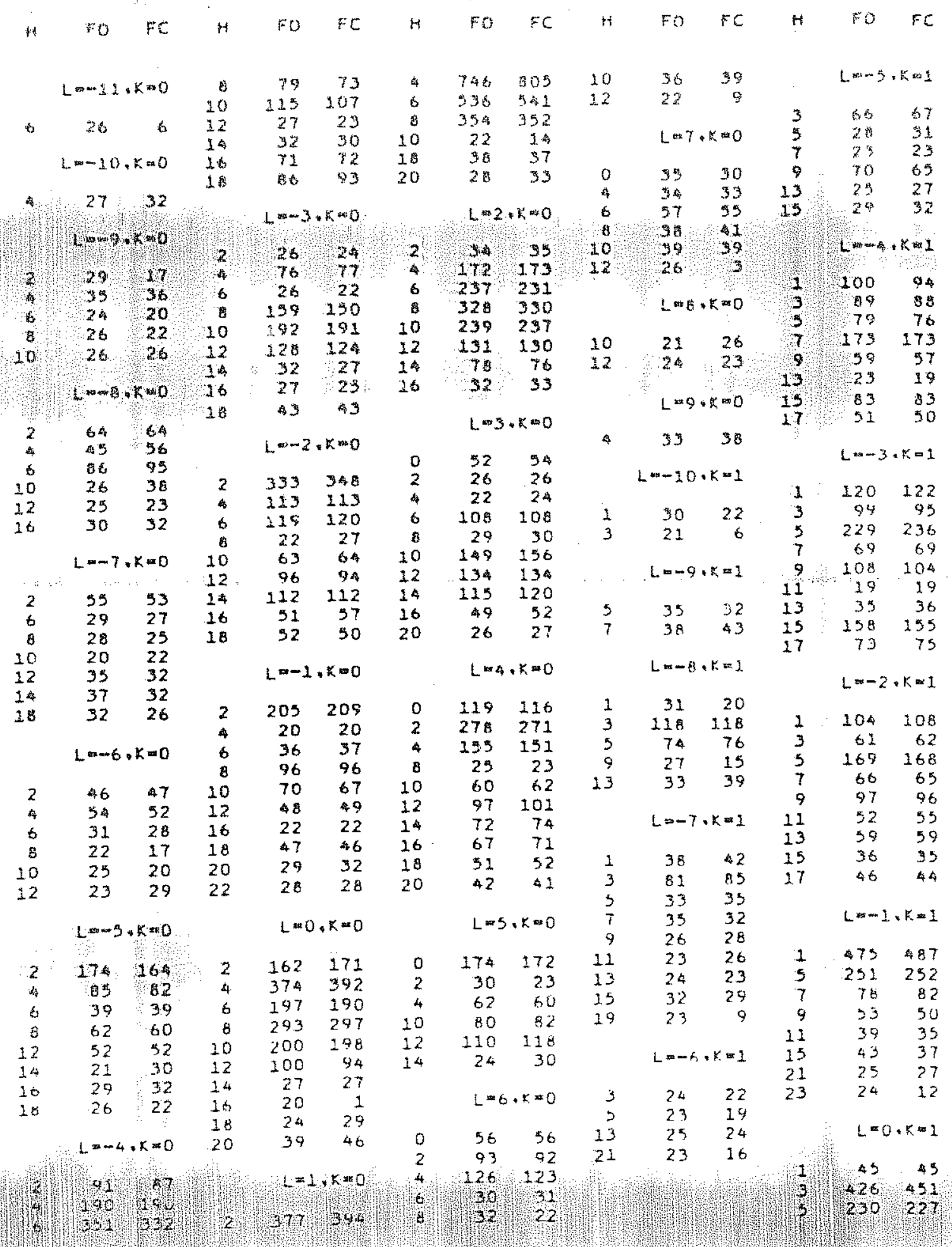




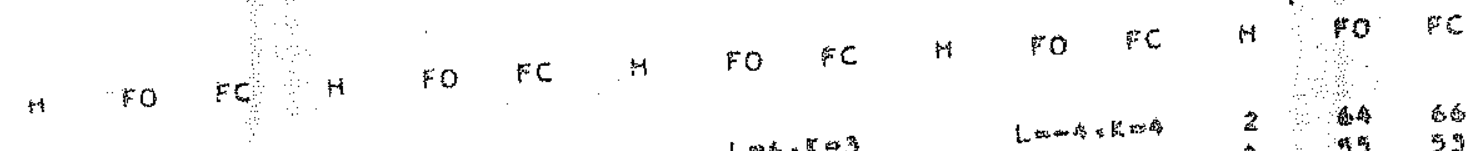

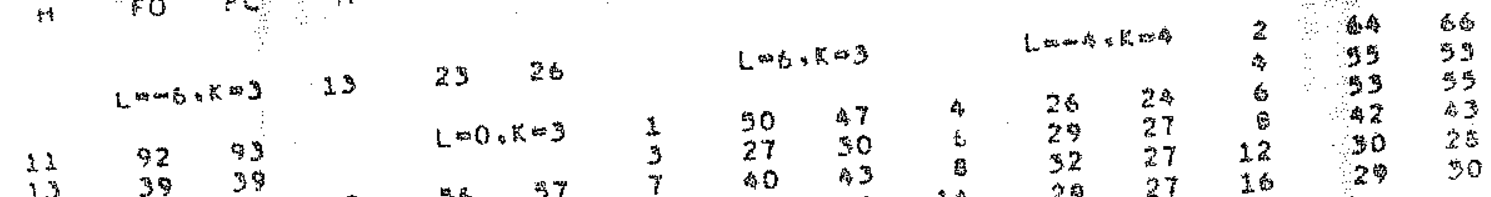

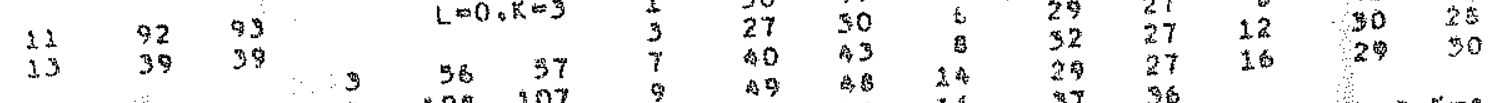

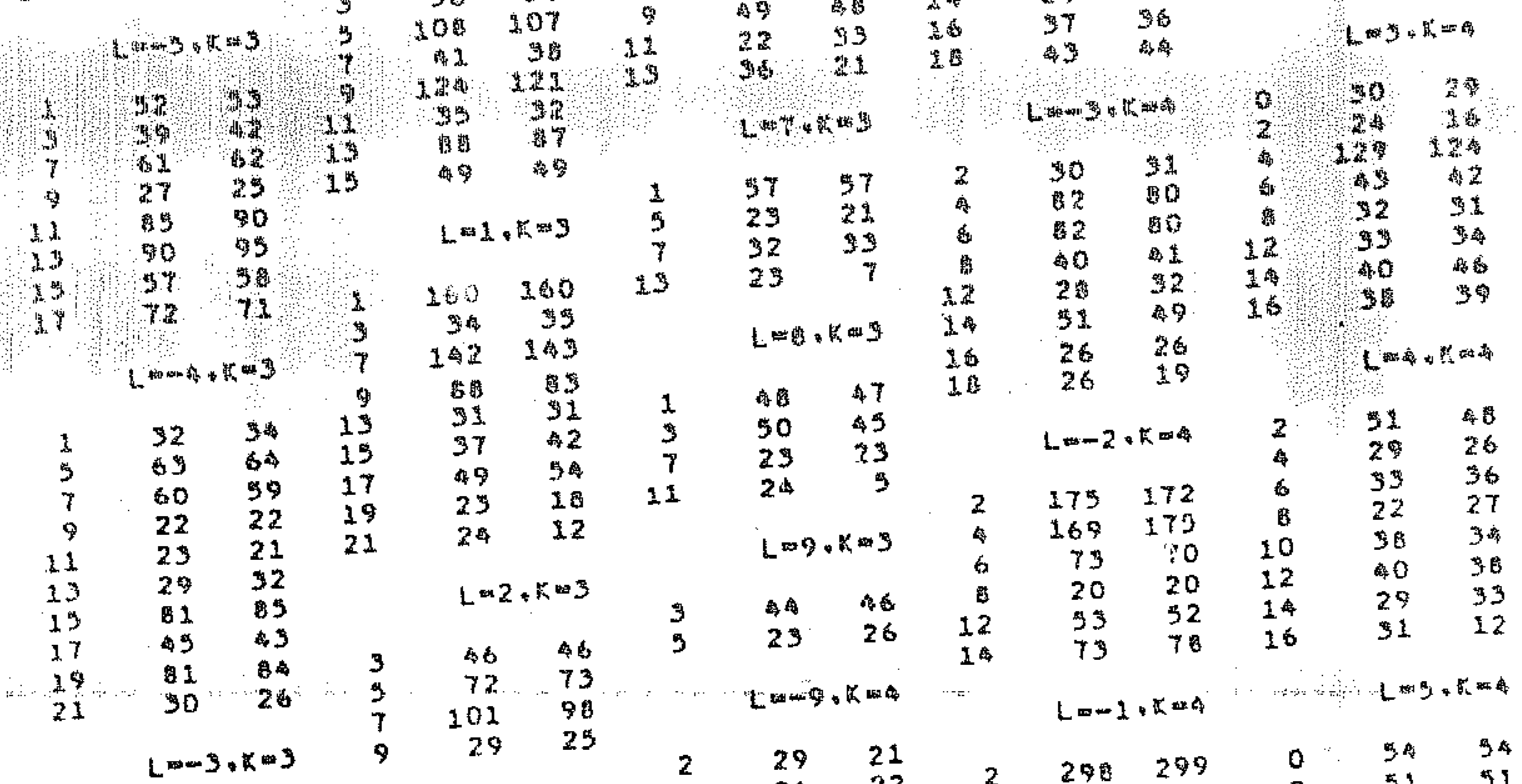

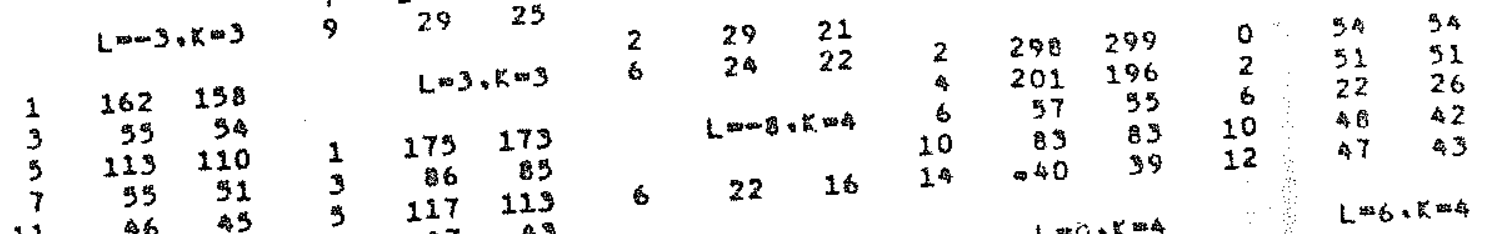

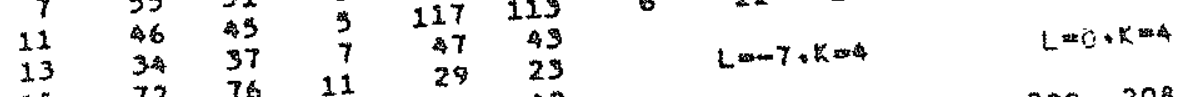

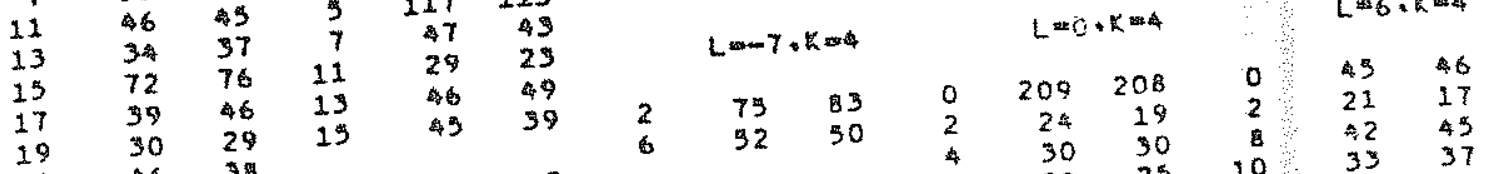

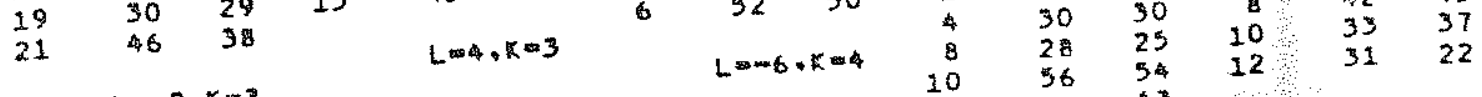

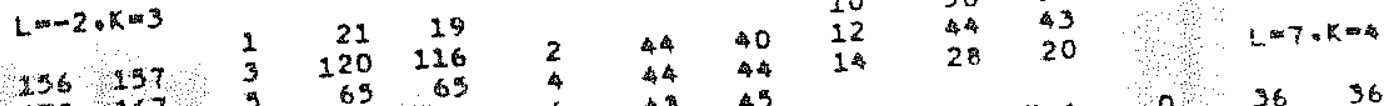

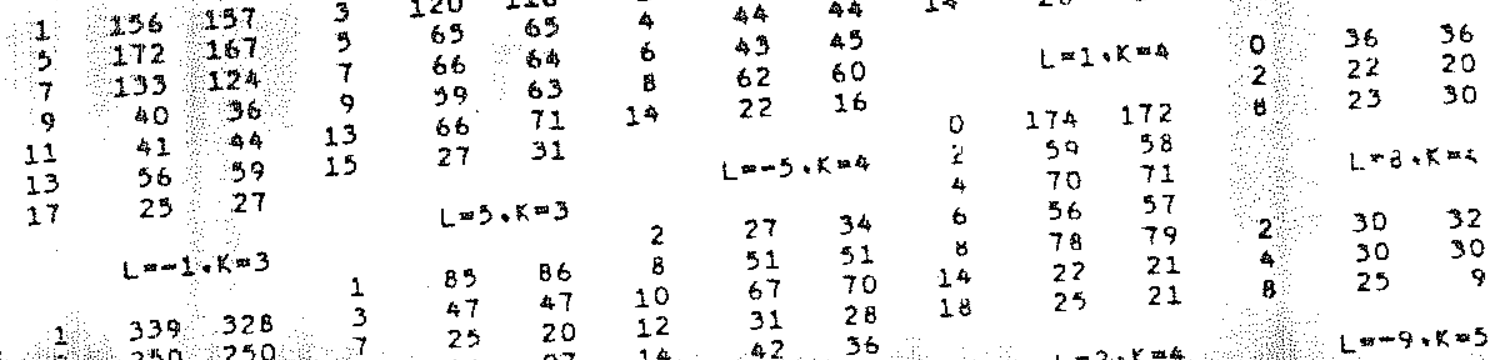

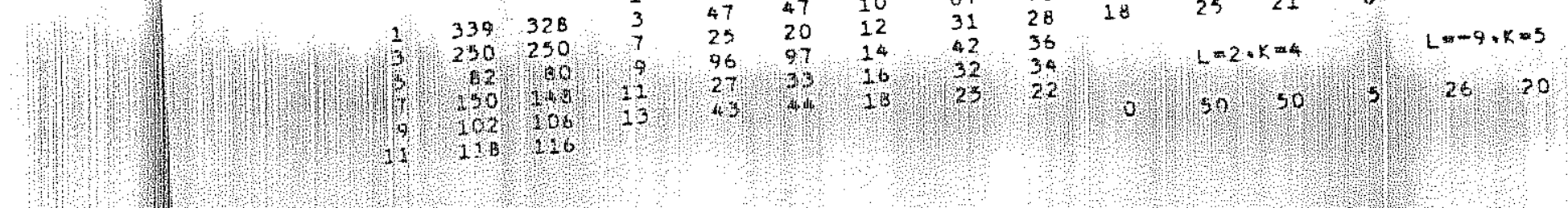




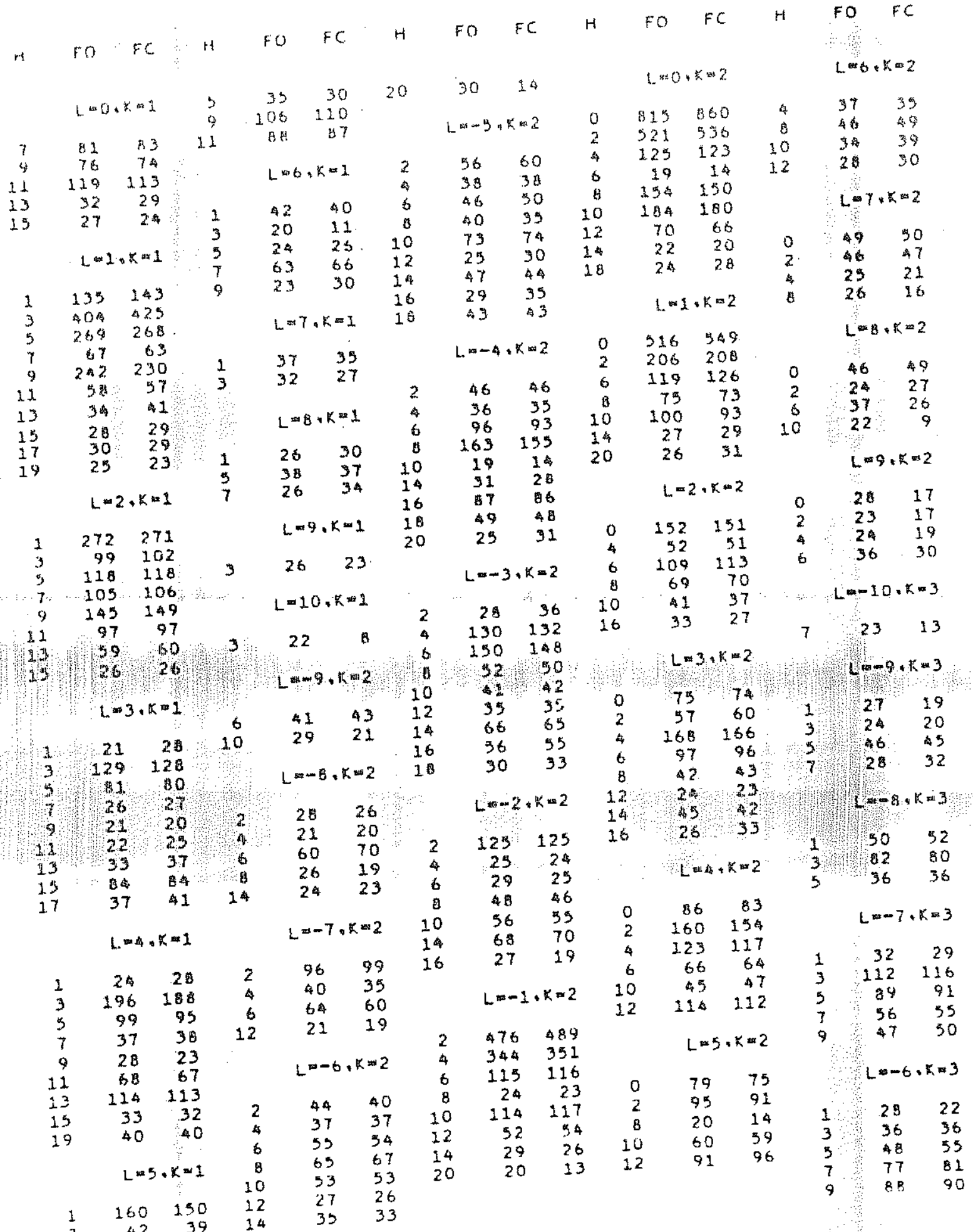


H FO FC M FO FC H FO FC H FO FC H F

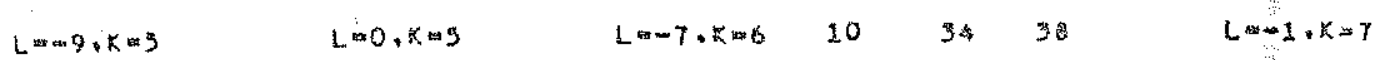

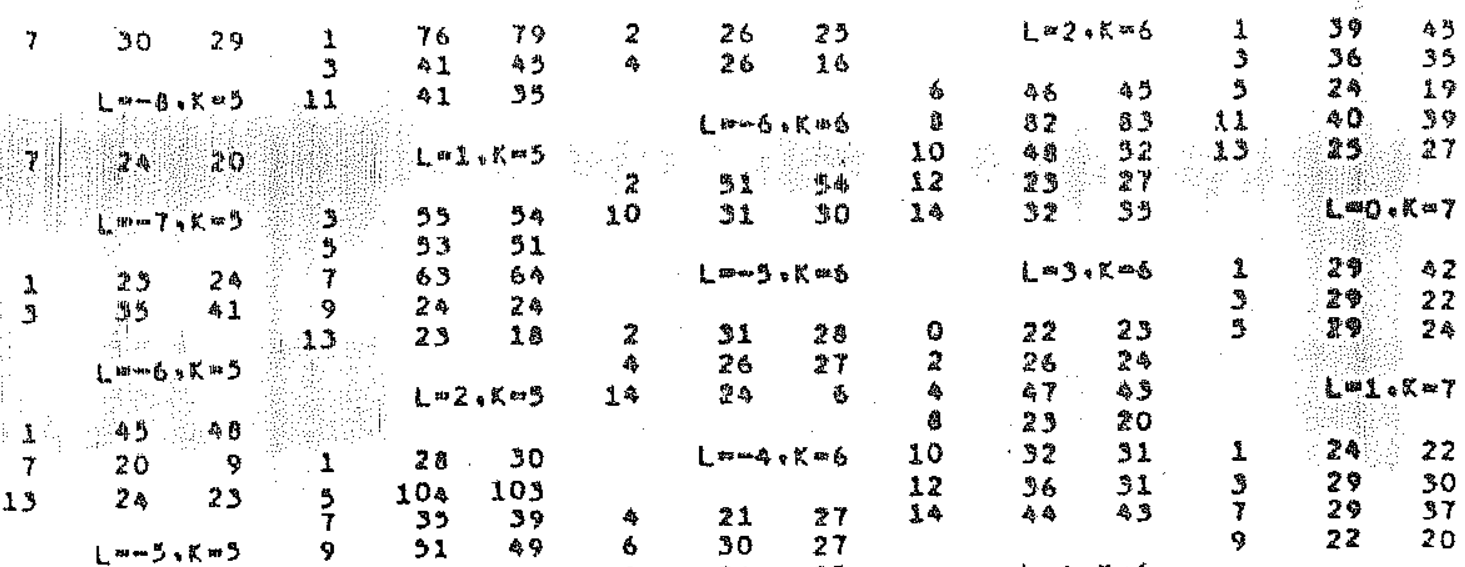

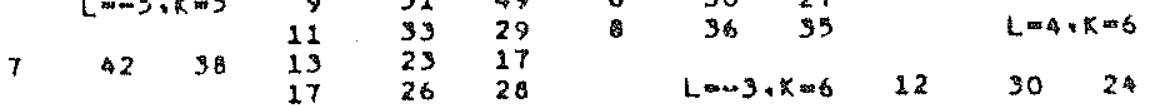
$L=-4, K=3$

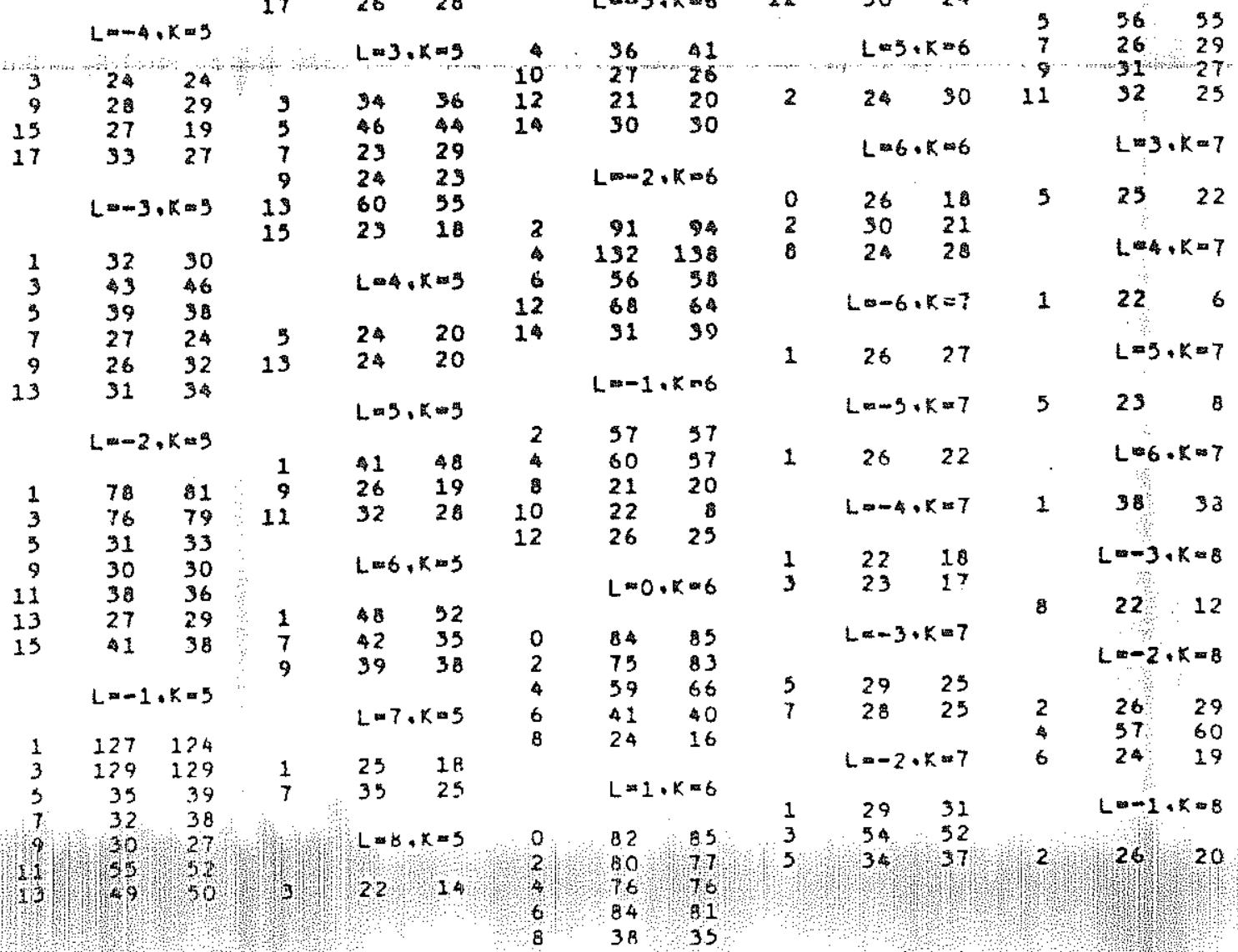


d

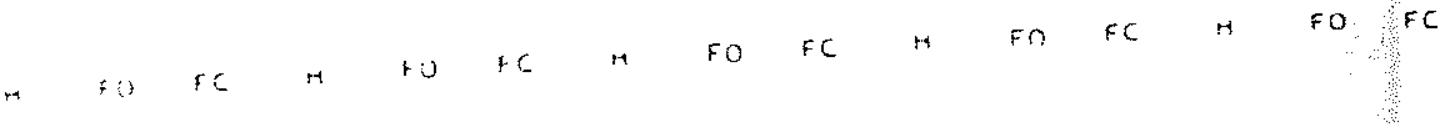

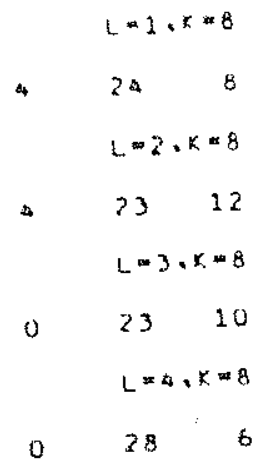

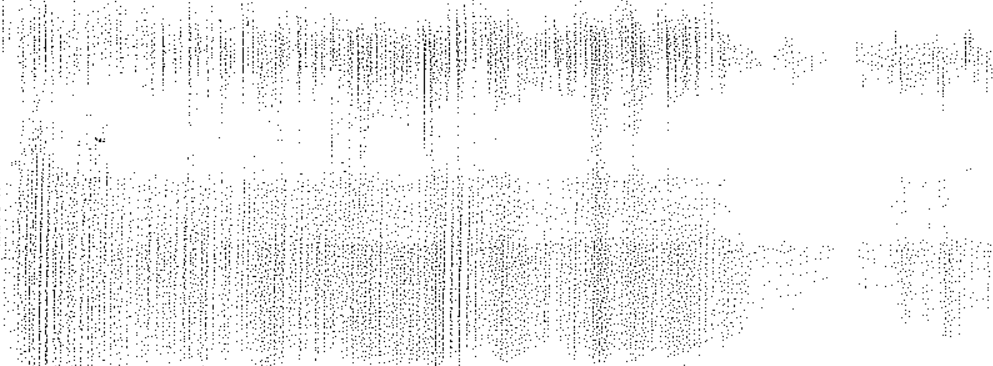

